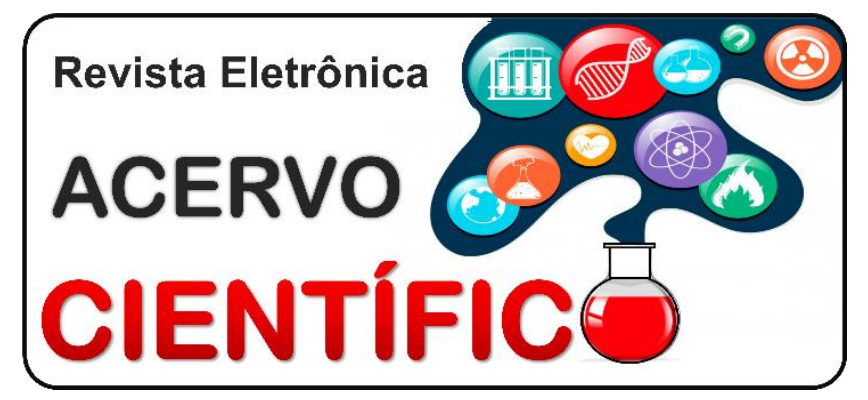

REVISÃO BIBLIOGRÁFICA

Recebido em: 11/2020

Aceito em: $12 / 2020$

Publicado em: 1/2021

\title{
Amamentação na prevenção do câncer de mama: revisão de literatura
}

\author{
Breastfeeding in breast cancer prevention: literature review \\ Lactancia en la prevención del cáncer de mama: revisión de la literatura
}

Fernanda Odete Souza Rodrigues ${ }^{1 *}$, João Vítor Flores Silveira1, Marina Santos Costa1, Giovanna Nogueira Torres ${ }^{1}$, Iwens Moreira de Faria ${ }^{2}$.

\begin{abstract}
Resumo: Esse artigo objetivou analisar estudos publicados sobre a influência da prática da amamentação na redução do risco da neoplasia mamária. Os artigos selecionados foram pesquisados nas bases de dados PubMed, LILACS, Scielo e Google Acadêmico e publicados entre os anos 2002 e 2020. Pouco tem sido estudada a relação entre câncer de mama e amamentação. É possível que a sucção da mama, realizada pelo recémnascido, promova alterações hormonais que repercutam sobre o desenvolvimento dessa doença. Entre os 11 artigos revisados, 10 atestaram o papel da amamentação na redução do surgimento de câncer de mama, tendo esta redução sido maior quanto mais longo o tempo da oferta de leite materno. Apesar dos inúmeros benefícios, as taxas de aleitamento materno no Brasil ainda são insuficientes. Tendo em vista o impacto positivo dessa prática sobre a redução da incidência do câncer de mama, orientações às gestantes sobre a relevância e o manejo desse processo se fazem necessárias.
\end{abstract}

Palavras-chave: Câncer de mama, Aleitamento materno, Lactação.

\begin{abstract}
This article aimed to analyze published studies on the influence of breastfeeding in reducing the risk of breast cancer. The selected articles were searched in the PubMed, LILACS, Scielo and Google Scholar databases and published between 2002 and 2020. Little has been studied about the relationship between breast cancer and breastfeeding. It is possible that breast sucking, performed by the newborn, promotes hormonal changes that affect the development of this disease. Among the 11 reviewed articles, 10 attested to the role of breastfeeding in reducing the onset of breast cancer, with this reduction being greater the longer the time of breast milk supply. Despite the numerous benefits, breastfeeding rates in Brazil are still insufficient. In view of the positive impact of this practice on reducing the incidence of breast cancer, guidance to pregnant women on the relevance and management of this process is necessary.
\end{abstract}

Keywords: Breast cancer, Breastfeeding, Lactation.

Resumen: Este artículo tuvo como objetivo analizar los estudios publicados sobre la influencia de la lactancia materna en la reducción del riesgo de cáncer de mama. Los artículos seleccionados fueron buscados en las bases de datos PubMed, LILACS, Scielo y Google Scholar y publicados entre 2002 y 2020 . Poco se ha estudiado sobre la relación entre el cáncer de mama y la lactancia materna. Es posible que la succión del pecho, realizada por el recién nacido, promueva cambios hormonales que inciden en el desarrollo de esta enfermedad. Entre los 11 artículos revisados, 10 atestiguan el papel de la lactancia materna en la reducción de la aparición del cáncer de mama, siendo esta reducción mayor cuanto mayor es el tiempo de suministro de leche materna. A pesar de los numerosos beneficios, las tasas de lactancia materna en Brasil siguen siendo insuficientes. En vista del impacto positivo de esta práctica en la reducción de la incidencia de cáncer de mama, es necesario orientar a las embarazadas sobre la relevancia y el manejo de este proceso.

Palabras clave: Cáncer de mama, Amamantamiento, Lactancia.

${ }^{1}$ Universidade de Itaúna (UIT), Itaúna - MG. *E-mail: nandasouzarodrigues@hotmail.com

2 Universidade Federal de Minas Gerais (UFMG), Belo Horizonte - MG. 


\section{INTRODUÇÃO}

O câncer é uma condição patológica caracterizada pela proliferação desordenada de células nos tecidos decorrente de modificações do código genético. A prevalência dessa enfermidade crônica tem aumentado mundialmente, sobretudo como consequência das transformações globais do estilo de vida da população e do aumento da expectativa de vida (OLIVEIRA ALR, et al., 2019). Sob esse aspecto, o carcinoma mamário é o mais frequentemente diagnosticado no sexo feminino, impactando 2,1 milhões de mulheres por ano (OMS, 2018).

Segundo dados fornecidos pela Organização Mundial da Saúde (OMS), em 2018, cerca de 627.000 mulheres morreram devido ao câncer de mama, o equivalente a $15,00 \%$ dos casos de morte por câncer entre pacientes do sexo feminino. No Brasil, o panorama é semelhante, tendo sido a estimativa da incidência de câncer de mama feminino no país para o ano de 2019 de, aproximadamente, 59.700 casos. Ressalta-se, entretanto, que esse registro não se distribuiu homogeneamente por todo o território nacional (INCA, 2019).

Diante desse contexto, faz-se necessário compreender os fatores que expõem as mulheres às maiores chances de adquirir câncer de mama. Além do sexo feminino, a idade é um importante aspecto, visto que a incidência aumenta, sobretudo, após os 35 anos (SANTOS TA e GONZAGA MFN, 2018). Fatores genéticos e hereditários, associados à grande densidade de tecido mamário, também contribuem para o desenvolvimento da doença. Sob o ponto de vista das causas potencialmente modificáveis, são relatadas na literatura a exposição excessiva às radiações ionizantes antes dos 40 anos, tabagismo, obesidade, sedentarismo, nuliparidade e ausência ou curtos períodos de amamentação (BUSHATSKY M, 2015).

Como fatores protetivos contra os tumores de mama ressaltam-se a amamentação em tempo adequado, a prática de exercícios físicos, alimentação saudável e manutenção do peso corporal. Tais fatores podem contribuir para a redução em até $30 \%$ do risco do surgimento de câncer de mama, demonstrando a importância de iniciativas individuais para a prevenção de agravos da saúde. Também corroboram para o manejo correto do câncer de mama a detecção precoce da doença por meio da observação de eventuais sinais e sintomas e o cumprimento da realização de exames de triagem, tais como a mamografia, em população de risco (SOARES JC, et al., 2019).

A influência do aleitamento materno na prevenção do câncer de mama merece destaque, visto que essa relação ainda é pouco discutida. Os benefícios da amamentação na saúde da mãe já são consolidados no meio científico e incluem o maior espaçamento intergestacional, além da aceleração na involução uterina, com consequente diminuição do sangramento pós-parto. Em relação à saúde do recém-nascido, por sua vez, a amamentação figura como grande aliada para a redução dos índices de mortalidade infantil ao proporcionar a produção de anticorpos e o desenvolvimento de processos cognitivos e motores (SOARES JC, et al., 2019).

Não por acaso, as recomendações da OMS, endossadas pelo Ministério da Saúde, orientam que o aleitamento materno deva ser realizado por dois anos ou mais, sendo prioritariamente exclusivo nos primeiros 6 meses. Apesar disso, sabe-se que apenas pouco mais da metade $(52,10 \%)$ das crianças brasileiras de até 24 meses receberam aleitamento materno em algum momento de sua vida. Além disso, ao se analisar o percentual de crianças amamentadas entre os 12 a 14 meses e dos 21 a 23 meses, os dados são ainda menores: 45,40\% e 31,80\%, respectivamente (MINISTÉRIO DA SAÚDE, 2015).

Estudos mais recentes mostraram que os altos índices de tumores de mama nos últimos anos estão intimamente relacionados à queda na taxa de natalidade e, consequentemente, à redução no período de lactação. Contribuem para essas mudanças o aumento do ingresso da mulher no mercado de trabalho, o retorno precoce ao emprego, bem como transformações no modo de vida que levam, por sua vez, à escolha de prioridades que não incluem a maternidade (CORDERO MJA, et al., 2010).

O cenário se torna preocupante na medida em que se considera o sucesso da prática da amamentação como fator de prevenção primária do câncer de mama, podendo ser responsável por $2 / 3$ da redução estimada da doença. Por essa razão, o fomento à oferta e manutenção do aleitamento materno pelo tempo 
indicado assume grande importância e se consolida como meta a ser alcançada (SOARES JC, et al., 2019; MINISTÉRIO DA SAÚDE, 2015).

Diante desse contexto e tendo em vista a importância da amamentação para a saúde do binômio materno-fetal, o presente estudo objetivou analisar trabalhos científicos publicados até o momento atual, com enfoque especial naqueles que avaliaram o papel do aleitamento materno na prevenção do câncer de mama. Além disso, este artigo visou, dada a precariedade de estudos encontrados na literatura, fomentar a elaboração de novas pesquisas sobre a temática em questão.

\section{MÉTODOS}

Os artigos utilizados nesta revisão bibliográfica foram pesquisados nas bases de dados National Library of Medicine (PubMED), Scientific Electronic Library Online (SciELO), Literatura Latino-americana e do Caribe em Ciências da Saúde (LILACS) e Google Acadêmico, e publicados entre os anos 2002 e 2020. Os descritores utilizados na busca foram: "câncer de mama", "aleitamento materno", "lactação", "breast feeding" e "breast cancer".

Em relação aos critérios de inclusão, foram selecionados os artigos que apresentavam dados originais e secundários sobre a associação entre câncer de mama e aleitamento materno. Quanto aos critérios de exclusão, foram desconsiderados trabalhos em duplicidade, publicados anteriormente ao ano 2002 e aqueles que, apesar de possuírem os descritores elegidos, não abordavam a temática da pesquisa diretamente. Após a aplicação dos critérios, foram selecionados 11 artigos, conforme demonstrado pelo fluxograma a seguir (Figura 1).

Figura 1 - Fluxograma da seleção dos estudos

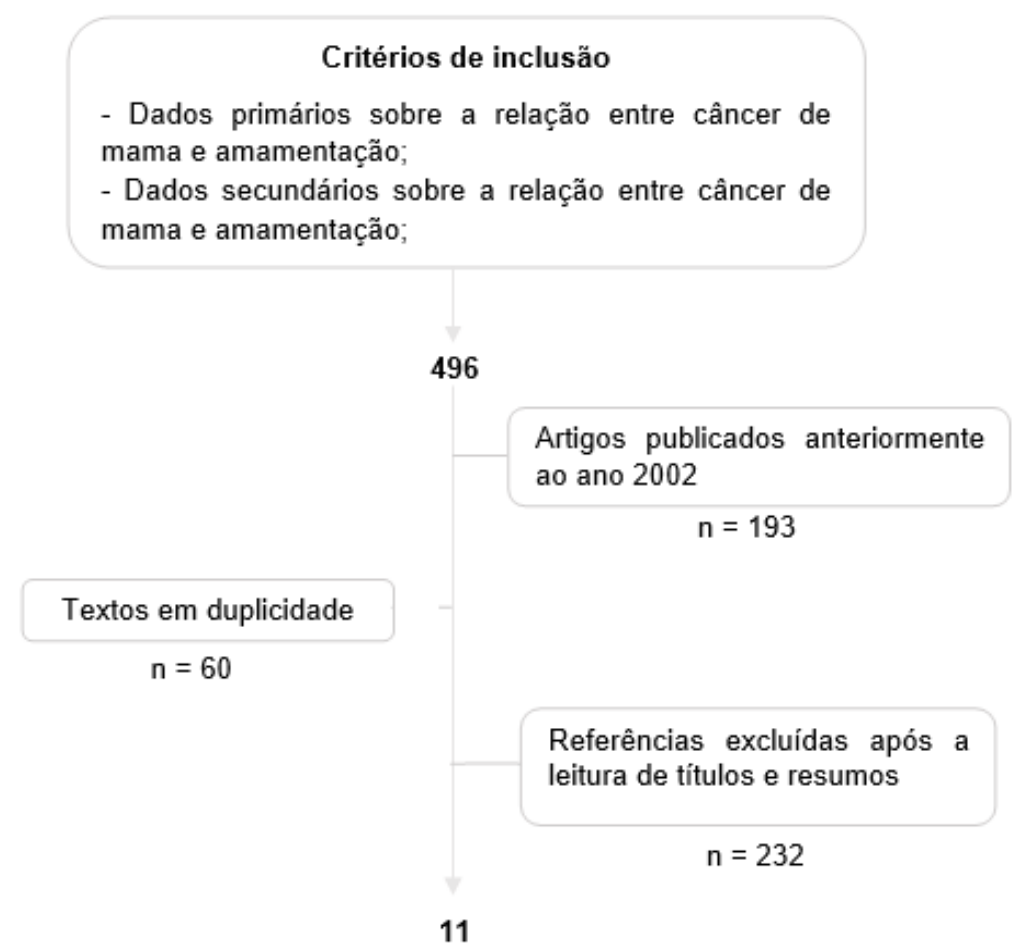

Fonte: RODRIGUES FOS, et al., 2020.

\section{RESULTADOS}

Dentre os 11 artigos analisados, publicados entre os anos de 2002 e 2019, apenas 2 representaram o cenário brasileiro, sendo o restante composto por publicações internacionais. O quadro 1 apresenta as principais características dos estudos selecionados (Quadro 1). 
Quadro 1 - Caracterização dos artigos com relação à associação entre câncer de mama e aleitamento materno.

\begin{tabular}{|c|c|c|c|}
\hline Ano & Autores & Revista & Principais resultados \\
\hline 2002 & $\begin{array}{l}\text { Grupo Colaborativo sobre } \\
\text { Fatores Hormonais no } \\
\text { Câncer de Mama }\end{array}$ & The Lancet & $\begin{array}{c}\text { O risco relativo de câncer de mama diminuiu } 4,3 \% \\
\text { a cada } 12 \text { meses de amamentação, além de uma } \\
\text { diminuição de } 7,0 \% \text { para cada nascimento. A } \\
\text { incidência cumulativa de câncer de mama nos } \\
\text { países desenvolvidos seria reduzida em mais da } \\
\text { metade, de } 6,3 \text { para } 2,7 \text { por } 100 \text { mulheres aos } 70 \\
\text { anos, se a oferta de leite materno ocorresse da } \\
\text { forma correta. }\end{array}$ \\
\hline 2008 & Huo D, et al. & $\begin{array}{l}\text { British Journal of } \\
\text { Cancer }\end{array}$ & $\begin{array}{c}\text { O risco diminuiu em } 5 \% \text { a cada nascimento } \\
\text { adicional após o primeiro e } 7 \% \text { a cada } 12 \text { meses } \\
\text { após a amamentação. Estima-se que a incidência } \\
\text { de câncer de mama tenha aumentado em 15\% } \\
\text { devido a menor duração da amamentação por } \\
\text { criança. }\end{array}$ \\
\hline 2010 & Coimbra R, et al. & $\begin{array}{c}\text { Revista Saúde } \\
\text { Coletiva }\end{array}$ & $\begin{array}{l}\text { O tempo de aleitamento materno complementar e } \\
\text { o tempo de aleitamento materno exclusivo não } \\
\text { apresentaram associação estatisticamente } \\
\text { significante para o desenvolvimento do câncer de } \\
\text { mama. }\end{array}$ \\
\hline 2010 & Cordero MJA, et al. & $\begin{array}{l}\text { Nutrición } \\
\text { Hospitalaria }\end{array}$ & $\begin{array}{c}\text { Houve correlação significativa entre idade do } \\
\text { diagnóstico do câncer, tempo de amamentação e } \\
\text { existência de histórico familiar e pessoal de } \\
\text { câncer. Não houve diferenças estatisticamente } \\
\text { significativas entre a idade média no diagnóstico } \\
\text { do câncer e a paridade. }\end{array}$ \\
\hline 2012 & $\begin{array}{c}\text { do Carmo França-Botelho } \\
\text { A, et al. }\end{array}$ & $\begin{array}{l}\text { Asian Pacific } \\
\text { Journal of } \\
\text { Cancer } \\
\text { Prevention }\end{array}$ & $\begin{array}{l}\text { Mães que não amamentam têm mostrado aumento } \\
\text { no risco de apresentaram cânceres reprodutivos. } \\
\text { Amamentar por longos períodos resultam, } \\
\text { estatisticamente, em reduções no risco de } \\
\text { desenvolver câncer de mama. }\end{array}$ \\
\hline 2015 & Águila YCR, et al. & $\begin{array}{l}\text { Revista Ciências } \\
\text { Médicas de Pinar } \\
\text { del Rio }\end{array}$ & $\begin{array}{c}\text { O câncer de mama prevaleceu entre mulheres } \\
\text { com } 50 \text { e } 69 \text { anos. Nenhuma oferta ou menos de } 4 \\
\text { meses de aleitamento materno foi um dos fatores } \\
\text { de risco mais frequentes para a neoplasia } \\
\text { mamária. }\end{array}$ \\
\hline 2015 & Navarro-lbarra MJ, et al. & $\begin{array}{l}\text { Nutrición } \\
\text { Hospitalaria }\end{array}$ & $\begin{array}{c}\text { Foi encontrada redução no risco de falecimentos } \\
\text { por neoplasias nas mulheres que amamentaram } \\
\text { em comparação com as que nunca } \\
\text { proporcionaram leite materno aos seus filhos. }\end{array}$ \\
\hline 2017 & Pérez-Escamilla R & $\begin{array}{l}\text { Sociedade } \\
\text { Brasileira de } \\
\text { Pediatria }\end{array}$ & $\begin{array}{l}\text { A inadequação da amamentação pode resultar em } \\
\text { cerca de } 5 \text { mil casos de câncer de mama, bem } \\
\text { como aproximadamente } 733 \text { bilhões de dólares em } \\
\text { custos relacionados à doença nos EUA. }\end{array}$ \\
\hline 2019 & Jelly P, Choudhary S. & $\begin{array}{l}\text { IP International of } \\
\text { Medical } \\
\text { Pediatrics and } \\
\text { oncology }\end{array}$ & $\begin{array}{c}\text { A amamentação acima dos } 6 \text { meses } \\
\text { recomendados pode prover proteção extra contra } \\
\text { o câncer de mama. Mulheres que amamentaram } \\
\text { têm menos risco de desenvolver câncer de mama } \\
\text { pré e pós-menopausa. }\end{array}$ \\
\hline 2019 & Ghalib HHA, et al. & $\begin{array}{l}\text { Journal of Family } \\
\text { Medicine and } \\
\text { Primary Care }\end{array}$ & $\begin{array}{c}\text { IMC } \geq 25 \mathrm{~kg} / \mathrm{m}^{2} \text { é fator de risco para câncer de } \\
\text { mama, enquanto exercício físico, amamentação } \\
\text { por mais de } 48 \text { meses e paridade maior que } 2 \\
\text { filhos têm efeito protetivo. }\end{array}$ \\
\hline 2019 & Fortner RT, et al. & $\begin{array}{l}\text { Breast Cancer } \\
\text { Research }\end{array}$ & $\begin{array}{c}\text { O risco de câncer de mama foi sugestivamente } \\
\text { maior entre as mulheres com maior paridade que } \\
\text { nunca amamentaram; associações foram nulas } \\
\text { entre as que já amamentaram. }\end{array}$ \\
\hline
\end{tabular}

Fonte: Rodrigues FOS, et al., 2020. 
Os resultados encontrados e interpretados, uma vez que pouco abrangem o contexto brasileiro, podem evidenciar realidades não condizentes com a do país. No entanto, se tratam de artigos de relevância mundial e com grande validação científica, pois baseiam-se em estudos de caso controle duplo cego, revisão de relatos clínicos, estudos transversais e ensaios clínicos.

De acordo com o quadro acima, dos 11 artigos listados, 3 demonstram que há, a cada 12 meses de amamentação, aproximadamente $5 \%$ de redução de chance do desenvolvimento de câncer de mama. Além disso, os trabalhos afirmaram que, a cada nascimento adicional, o risco do surgimento de neoplasia mamária na mulher reduz de 5\% a 7\% (HUO D, et al., 2008; COLLABORATIVE GROUP ON HORMONAL FACTORS IN BREAST CANCER, 2002).

Ademais, as pesquisas foram unânimes ao demonstraram que mulheres com pouca ou nenhuma oferta de aleitamento materno estiveram mais suscetíveis à probabilidade de desenvolver o câncer de mama. Ainda de acordo com esses estudos, foi observado que o aumento no surgimento da doença aproximou-se de $15 \%$ nos grupos que não ofertaram ou ofereceram leite materno por pouco tempo (DO CARMO FRANÇA-BOTELHO A, 2012; ÁGUILA YCR, 2015).

Nesse aspecto, trabalhos que encontraram correlação positiva entre a neoplasia mamária e o câncer de mama ressaltaram a relevância da manutenção da oferta de leite materno por, no mínimo, 6 meses. Idealmente, no entanto, apontaram a relevância e eficácia ainda mais significativa da amamentação quando praticada por, ao menos, um ano, sendo esta capaz de reduzir a incidência de câncer de mama em, aproximadamente, 48\% (SOARES JC, et al., 2019; HUO D, et al., 2008).

Mediante análise sistemática dos estudos, observou-se que apenas um deles sugeriu a inexistência da relação entre o surgimento do câncer de mama e o tempo de aleitamento materno. Para tanto, os autores indicaram que não havia sido comprovada associação estatística significativa entre o número de meses de aleitamento materno e o surgimento da neoplasia maligna (COIMBRA R, et al., 2010).

\section{DISCUSSÃO}

A literatura sobre os benefícios da amamentação para a saúde da mulher é escassa. No entanto, a maior parte das publicações nacionais e internacionais realizadas até o presente momento relataram a existência de associação entre a amamentação e a menor incidência de doenças como os cânceres de mamas e ovários, além de diminuir a predisposição às patologias ósseas. Pesquisas têm apontado, todavia, que a maior parte das informações sobre o aleitamento materno nas consultas de pré-natal são voltadas à saúde do recém-nascido, sendo as mães poupadas de orientações primordiais para a promoção de sua própria saúde (LEDESMA ER, et al., 2019; GHALIB HHA, et al., 2019).

Em consonância com a literatura, os estudos selecionados para análise nesta revisão bibliográfica referiram a responsabilidade de amamentação na redução de aproximadamente $70 \%$ da probabilidade de a mulher desenvolver câncer de mama, principalmente se a prática for mantida de forma exclusiva por cerca de 73 a 108 meses durante a vida da mulher Sustentam essa afirmativa as pesquisas que concluíram que 0 aleitamento materno produz alterações nos níveis de hormônios sexuais femininos, provendo efeito protetor à lactante (NAVARRO-IBARRA MJ, et al., 2015; ÁGUILA YCR, et al., 2015).

Sob esse aspecto, com relação ao mecanismo fisiopatológico que leva ao desenvolvimento do câncer de mama, é consenso que alguns carcinomas mamários possuem receptores para estrogênio e progesterona. Assim, quando a criança realiza sucção da mama, há estimulação do aumento da produção de prolactina que, por meio de sua atuação no hipotálamo, reduz a pulsatilidade desses hormônios (JELLY $P$ e CHOUDHARY S, 2019). Dessa forma, os receptores tumorais não são ativados e ocorre diminuição na neoformação de células carcinogênicas, razão pela qual a amamentação tem sido considerada importante estratégia de saúde (MINISTÉRIO DA SAÚDE, 2015).

Outros fatores protetores contra o câncer de mama, em associação com a amamentação, foram apontados pelos trabalhos avaliados. A esse respeito, foi observado que mulheres que possuíam o componente genético para a neoplasia e que não ofertaram o leite materno por um período mínimo de 6 
meses adoeceram em idades precoces, sendo esta informação corroborada por outras publicações internacionais. Comparativamente, em lactantes que amamentaram por mais de 6 meses e que possuíam histórico familiar predisponente ao surgimento da neoplasia, o adoecimento ocorreu 17 anos mais tarde que no grupo anterior (CARMICHAEL H, 2017).

Houve controvérsias no que diz respeito a etapa da vida materna em que o aleitamento materno proporciona maior proteção contra o câncer de mama, levantando questionamentos se o efeito protetivo prevaleceria por toda a menacme ou apenas na menopausa. Pesquisas relataram que a proteção oferecida pela amamentação foi maior em mulheres na pré-menopausa, tendo também sido encontrada relação doseresposta entre número de meses de amamentação e menos chance de apresentar câncer de mama (LEDESMA ER, et al., 2019).

Apesar dos conhecidos benefícios sobre o aleitamento materno, foi constatado, ao longo dos anos, que a amamentação tem deixado de ser vista como um processo fisiológico natural da maternidade e se tornado uma opção que compete com outras de maior praticidade, como a nutrição por fórmulas ou leites infantis modificados. O uso de tais produtos, embora não altere o risco de desenvolvimento de câncer de mama, repercute sobre a diminuição do tempo de aleitamento materno exclusivo e, consequentemente, em seu efeito protetivo para a mãe (JELLY P e CHOUDHARY S, 2019).

Ainda que iniciativas como o Hospital Amigo da Criança (HAC) recomendem que fórmulas de leite sejam prescritas apenas após os 6 meses de idade, se necessário, e, preferencialmente, de forma exclusiva, a realidade brasileira é contraditória. Nesse sentido, pediatras pouco envolvidos no contexto do HAC podem impactar negativamente no comportamento da lactante ao induzir o desmame precoce. No mesmo estudo, puérperas referiram não terem iniciado a amamentação no centro obstétrico, mesmo nas maternidades com o título de Hospital Amigo da Criança, demonstrando a fragilidade desse sistema (UEMA RTB, et al., 2015).

Sobre esse assunto, foram encontrados na literatura grupos específicos de mulheres que demonstraram maior tendência ao desmame precoce. Nesse sentido, os autores apontaram que mães com idade menor que 20 anos, com poucas consultas pré-natal, que não convivem com o companheiro ou possuem família instável, com menor grau de escolaridade, primíparas e que trabalham fora ou sem licença maternidade possuem maiores taxas de abandono do aleitamento materno (UEMA RTB, et al., 2015; GHALIB HHA, 2019).

Além dos aspectos supracitados, dados abordaram, ainda, que há um padrão de aleitamento dependente da situação econômica. Em países desenvolvidos, por exemplo, as puérperas preferiram amamentar seus filhos com métodos artificiais, ao passo que nos países subdesenvolvidos houve predileção pelo aleitamento natural. Quando realizada a opção pelo aleitamento natural, por sua vez, o risco de surgimento do câncer de mama foi reduzido em quase $4,5 \%$ para cada ano em que se ofertou 0 aleitamento materno, tanto em países desenvolvidos quanto em países não desenvolvidos (LUBOLD AM, 2019).

Quanto ao impacto do aleitamento materno sobre os óbitos por câncer de mama, resultados encontrados reforçaram a relevância desta prática ao informar que, aproximadamente, 20.000 mortes foram evitadas quando a prática foi realizada no tempo correto. Foi estimado, ainda, que outras 20.000 mortes seriam prevenidas se a amamentação fosse estendida para 12 meses por criança em países desenvolvidos, como os Estados Unidos, e para 2 anos por criança em países de baixa e média renda (WAGNER LPB, et al., 2020).

No Brasil, apesar da implementação do Programa Nacional do Incentivo ao Aleitamento Materno em 1981 e de suas repercussões sobre o aumento no aleitamento materno, ainda são registradas taxas inferiores às preconizadas pelos órgãos de saúde. A prevalência de aleitamento materno exclusivo em crianças menores de 6 meses foi de $41 \%$, dado que mostra que mais da metade das crianças brasileiras não foram amamentadas de modo exclusivo no tempo recomendado. Além disso, a duração média da oferta de leite materno foi de 54 meses 11 dias (VICTORA CG, et al., 2016).

A análise de publicações brasileiras revelou que a insuficiência nas taxas de aleitamento materno, com consequente aumento na incidência da neoplasia mamária, pode ser atribuída à falta de informação 
prestada por profissionais da saúde nos diversos níveis de atenção. Os estudos relataram que, em média, $30,00 \%$ das lactantes entrevistadas informaram não terem sido orientadas sobre o efeito protetor da amamentação contra o carcinoma mamário, sendo este dado mais satisfatório do que encontrado por grupos de pesquisadores de outros países (SOUZA JCA, et al., 2019).

Diante desse contexto, pesquisas afirmaram que a capacitação profissional contribui, entre outros aspectos, para a superação de eventuais dificuldades inerentes à prática da amamentação. Relatos de produção insuficiente de leite, ingurgitamento mamário, pega incorreta e surgimento de fissuras mamárias dolorosas estiveram relacionados ao desmame precoce e seriam, possivelmente, solucionados com auxílio profissional adequado, ocasionando aumento na taxa de aleitamento materno e redução no risco de câncer de mama (VASCONCELOS TC, et al., 2020; PÉREZ-ESCAMILLA R, 2017).

\section{CONSIDERAÇÕES FINAIS}

Nos últimos anos, as pesquisas realizadas em diversos países referiram a importância da amamentação na diminuição da incidência do câncer de mama, sendo esta capaz de contribuir para a redução de aproximadamente $70 \%$ da probabilidade de a mulher desenvolver neoplasia mamária, principalmente se a prática for mantida de forma exclusiva pelo tempo preconizado pelos órgãos de saúde. Apesar disso, a inadequação do tempo de amamentação tem se apresentado com relevante prevalência e, por esse motivo, orientações às gestantes sobre o processo da amamentação e instrução de profissionais de saúde devem ser entendidos como ponto de partida para a resolução dessa problemática.

\section{REFERÊNCIAS}

1. ÁGUILA YCR, et al. Cáncer de mama, su caracterización epidemiológica. Rev Ciencias Médicas. 2015; 19(4): 619629.

2. BRASIL. Ministério da Saúde. Instituto Nacional de Câncer José Alencar Gomes da Silva (INCA). A situação do câncer de mama no Brasil: Síntese de dados dos sistemas de informação. Rio de Janeiro, RJ. 2019.

3. BRASIL Ministério da Saúde. Saúde da criança: Aleitamento Materno e Alimentação Complementar. Caderno de Atenção Básica. 2 ed. Brasília, DF. 2015.

4. BUSHATSKY M, et al. Educação em saúde: uma estratégia de intervenção frente ao câncer de mama. Ciência, Cuidado e Saúde. 2015; 14(1): 870-878.

5. CARMICHAEL $\mathrm{H}$, et al. Breast cancer screening of pregnant and breastfeeding women with BRCA mutations. Breast Cancer Res Treat. 2017; 162(2): 225-230.

6. COIMBRA R, et al. Fatores gineco-obstétricos associados à neoplasia maligna da mama em mulheres de 20 a 64 anos de idade. Saúde Coletiva, 2010; 7(39): 76-81

7. COLLABORATIVE GROUP ON HORMONAL FACTORS IN BREAST CANCER. Breast cancer and breastfeeding: collaborative reanalysis of individual data from 47 epidemiological studies in 30 countries, including 50.302 women with breast cancer and 96.973 women without the disease. The Lancet. 2002; 360(9328): 187-195.

8. CORDERO MJA, et al. Lactancia materna: un método eficaz en la prevención del cáncer de mama. Nutr Hosp. 2010; 25(6): 954-958.

9. DO CARMO FRANÇA-BOTELHO A, et al. Breastfeeding and its relationship with reduction of breast cancer: a review. Asian Pac J Cancer Prev. 2012; 13(11): 5327-5332.

10. GHALIB HHA, et al. Risk factors assessment of breast cancer among Iraqi Kurdish women: Case-control study. Journal of Family Medicine and Primary Care. 2019; 8(12): 3990-3997.

11. HUO D, et al. Parity and breastfeeding are protective against breast cancer in Nigerian women. Br J Cancer. 2008; 98(5): 992-996.

12. JELLY P, CHOUDHARY S. Breastfeeding and breast cancer: A risk reduction strategy. Int $J$ Med Paediatr Oncol. 2019; 5(2), 47-50.

13. LEDESMA ER, et al. Factores de riesgo del cáncer de mama en un consultorio de la Atención Primaria de Salud. Rev haban cienc méd. 2019; 18(2): 308-322.

14. LUBOLD AM. Historical-qualitative analysis of breastfeeding trends in three OECD countries. International Breastfeeding Journal. 2019; 14(36).

15. NAVARRO-IBARRA MJ, et al. Influence of reproductive factors, breastfeeding and obesity on the risk of breast cancer in mexican women. Nutricion Hospitalaria. 2015; 32(1):291-298. 
16. OLIVEIRA ALR, et al. Fatores de risco e prevenção do câncer de mama. Revista Cadernos de Medicina. 2019; 2(3): $135-145$.

17. PÉREZ-ESCAMILLA R. Breastfeeding in Brazil: major progress, but still a long way to go. J Pediatr (Rio J). 2017; 93(2): 107-110.

18. SANTOS TA, GONZAGA MFN. Fisiopatologia do câncer de mama e fatores relacionados. Revista Saúde em Foco. 2018; 10: 359-366.

19. SOARES JC, et al. Aleitamento materno na prevenção do câncer de mama: uma revisão integrativa da literatura. Revista Uningá, 2019; 56(6): 13-22.

20. SOUZA JCA, et al. Conhecimento das puérperas sobre os benefícios da amamentação em ambiente hospitalar. Cad da Esc de Saúde. 2019; 18(1):1-22.

21. UEMA RTB, et al. Prevalence and factors associated with breastfeeding in Brazil between the years 1998 and 2013 : a systematic review. Semina cienc. biol. saude. 2015; 36(1): 349-362

22. VASCONCELOS TC, et al. Fatores que interferem no aleitamento materno exclusivo durante os primeiros seis meses de vida do bebê. Revista Pró-UniverSUS. 2020; 11 (1): 80-87.

23. VICTORA CG, et al. Amamentação no século 21: epidemiologia, mecanismos, e efeitos ao longo da vida. Epidemiol Serv Saúde. 2016; 25(1): 1-24.

24. WAGNER LPB, et al. Strengthening and weakening factors for breastfeeding from the perspective of the nursing mother and her family. Rev. esc. enferm. USP. 2020; 54: e03563.

25. WORLD HEALTH ORGANIZATION. Breast Cancer. Genebra: WHO. Disponível em: <https://www.who.int/cancer/prevention/diagnosis-screening/breast-cancer/en/>. Acesso em: 27 de julho de 2020. 